\title{
Fourth order elliptic system with dirichlet boundary condition
}

Tacksun Jung $^{1^{*}}$ and Q-Heung Choi ${ }^{2}$

\author{
* Correspondence: tsjung@kunsan. \\ ac.kr \\ 'Department of Mathematics, \\ Kunsan National University, Kunsan \\ 573-701, Korea \\ Full list of author information is \\ available at the end of the article
}

\begin{abstract}
We investigate the multiplicity of the solutions of the fourth order elliptic system with Dirichlet boundary condition. We get two theorems. One theorem is that the fourth order elliptic system has at least two nontrivial solutions when $\lambda_{k}<c<\lambda_{k+1}$ and $\lambda_{k+n}\left(\lambda_{k+n}-c\right)<a+b<\lambda_{k+n+1}\left(\lambda_{k+n+1}-c\right)$. We prove this result by the critical point theory and the variation of linking method. The other theorem is that the system has a unique nontrivial solution when $\lambda_{k}<c<\lambda_{k+1}$ and $\lambda_{k}\left(\lambda_{k}-c\right)<0$, $a+b<$ $\lambda_{k+1}\left(\lambda_{k+1}-c\right)$. We prove this result by the contraction mapping principle on the Banach space.
\end{abstract}

AMS Mathematics Subject Classification: 35J30, 35J48, 35J50

Keywords: Fourth order elliptic system, fourth order elliptic equation, variation linking theorem, contraction mapping principle

\section{Introduction}

Let $\Omega$ be a smooth bounded region in $R^{n}$ with smooth boundary $\partial \Omega$. Let $\lambda_{1}<\lambda_{2} \leq \ldots$ $\leq \lambda_{k} \leq \ldots$ be the eigenvalues of $-\Delta$ with Dirichlet boundary condition in $\Omega$. In this paper we investigate the multiplicity of the solutions of the following fourth order elliptic system with Dirichlet boundary condition

$$
\begin{array}{lr}
\Delta^{2} u+c \Delta u=a\left((u+v+1)^{+}-1\right) & \text { in } \Omega, \\
\Delta^{2} v+c \Delta v=b\left((u+v+1)^{+}-1\right) & \text { in } \Omega, \\
u=0, v=0, \Delta u=0, \Delta v=0 & \text { on } \partial \Omega,
\end{array}
$$

where $c \in R, u^{+}=\max \{u, 0\}$ and $a, b \in R$ are constant. The single fourth order elliptic equations with nonlinearities of this type arises in the study of travelling waves in a suspension bridge ([6]) or the study of the static deflection of an elastic plate in a fluid and have been studied in the context of the second order elliptic operators. In particular, Lazer and McKenna [6] studied the single fourth order elliptic equation with Dirichlet boundary condition

$$
\begin{gathered}
\Delta^{2} u+c \Delta u=b\left((u+1)^{+}-1\right), \quad \text { in } \Omega, \\
u=0, \quad \Delta u=0 \quad \text { on } \partial \Omega .
\end{gathered}
$$

Tarantello [10] also studied problem (1.2) when $c<\lambda_{1}$ and $b \geq \lambda_{1}\left(\lambda_{1}-c\right)$. She show that (1.2) has at least two solutions, one of which is a negative solution. She obtained this result by degree theory. Micheletti and Pistoia [8] proved that if $c<\lambda_{1}$ and $b \geq \lambda_{2}$ $\left(\lambda_{2}-c\right)$, then (1.2) has at least four solutions by the Leray-Schauder degree theory.

\section{Springer}

(C) 2011 Jung and Choi; licensee Springer. This is an Open Access article distributed under the terms of the Creative Commons Attribution License (http://creativecommons.org/licenses/by/2.0), which permits unrestricted use, distribution, and reproduction in any medium, provided the original work is properly cited. 
Micheletti, Pistoia and Sacon [9] also proved that if $c<\lambda_{1}$ and $b \geq \lambda_{2}\left(\lambda_{2}-c\right)$, then (1.2) has at least three solutions by variational methods. Choi and Jung [2] also considered the single fourth order elliptic problem

$$
\begin{gathered}
\Delta^{2} u+c \Delta u=b u^{+}+s \quad \text { in } \Omega, \\
u=0, \quad \Delta u=0 \quad \text { on } \partial \Omega .
\end{gathered}
$$

They show that (1.3) has at least two nontrivial solutions when $c<\lambda_{1}, \lambda_{1}\left(\lambda_{1}-c\right)<b$ $<\lambda_{2}\left(\lambda_{2}-c\right)$ and $s<0$ or when $\lambda_{1}<c<\lambda_{2}, b<\lambda_{1}\left(\lambda_{1}-c\right)$ and $s>0$. They also obtained these results by using the variational reduction method. They [3] also proved that when $c<\lambda_{1}, \lambda_{1}\left(\lambda_{1}-c\right)<b<\lambda_{2}\left(\lambda_{2}-c\right)$ and $s<0$, (1.3) has at least three solutions by using degree theory. In [7-9] the authors investigate the existence of solutions of jumping problems with Dirichlet boundary condition.

In this paper we improve the multiplicity results of the single fourth order elliptic problem to that of the fourth order elliptic system. Our main results are as follows:

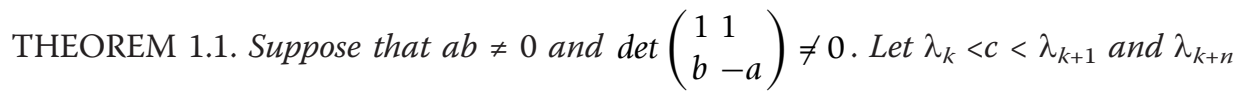
$\left(\lambda_{k+n}-c\right)<a+b<\lambda_{k+n+1}\left(\lambda_{k+n+1}-c\right)$. Then system (1.1) has at least two nontrivial solutions.

THEOREM 1.2. Suppose that $a b \neq 0$ and $\operatorname{det}\left(\begin{array}{ll}1 & 1 \\ b & -a\end{array}\right) \neq 0$. Let $\lambda_{k}<c<\lambda_{k+1}$ and $\lambda_{k}$ $\left(\lambda_{k}-c\right)<0, a+b<\lambda_{k+1}\left(\lambda_{k+1}-c\right)$. Then system (1.1) has a unique nontrivial solution.

In section 2 we define a Banach space $H$ spanned by eigenfunctions of $\Delta^{2}+c \Delta$ with Dirichlet boundary condition and investigate some properties of system (1.1). In section 3, we prove Theorem 1.1 by using the critical point theory and variation of linking method. In section 4 , we prove Theorem 1.2 by using the contraction mapping principle.

\section{Fourth order elliptic system}

The eigenvalue problem $\Delta^{2} u+c \Delta u=\mu u$ in $\Omega$ with $u=0, \Delta u=0$ on $\partial \Omega$ has also infinitely many eigenvalues $\mu_{k}=\lambda_{k}\left(\lambda_{k}-c\right), k \geq 1$ and corresponding eigenfunctions $\varphi_{k}, k \geq 1$. We note that $\lambda_{1}\left(\lambda_{1}-c\right)<\lambda_{2}\left(\lambda_{2}-c\right) \leq \lambda_{3}\left(\lambda_{3}-c\right)<\ldots$

The system

$$
\begin{array}{lr}
\Delta^{2} u+c \Delta u=a\left((u+v+1)^{+}-1\right) & \text { in } \Omega, \\
\Delta^{2} v+c \Delta v=b\left((u+v+1)^{+}-1\right) & \text { in } \Omega, \\
u=0, v=0, \Delta u=0, \Delta v=0 & \text { on } \partial \Omega
\end{array}
$$

can be transformed to the equation

$$
\begin{gathered}
\Delta^{2}(u+v)+c \Delta(u+v)=(a+b)\left((u+v+1)^{+}-1\right) \quad \text { in } \Omega, \\
u=0, v=0, \quad \Delta u=0, \quad \Delta v=0 \quad \text { on } \partial \Omega .
\end{gathered}
$$

We also have

$$
\begin{array}{cl}
\Delta^{2}(b u-a v)+c \Delta(b u-a v)=0 & \text { in } \Omega, \\
u=0, v=0, \quad \Delta u=0, \quad \Delta v=0 & \text { on } \partial \Omega .
\end{array}
$$

It follows from the above equation that $b u-a v=0$. If $u+v=w$ is a solution of (2.1), then the system 


$$
\begin{aligned}
& u+v=w, \\
& b u-a v=0
\end{aligned}
$$

has a unique solution of (1.1) since $\operatorname{det}\left(\begin{array}{ll}1 & 1 \\ b & -a\end{array}\right) \neq 0$. Hence the number of the solutions $w=u+v$ of (1.1) is equal to that of (2.1). To investigate the multiplicity of (1.1) it is enough to find the multiplicity of (2.1). Let us set $w=u+v$. Then (2.1) is equivalent to the equation

$$
\begin{gathered}
\Delta^{2} w+c \Delta w=(a+b)\left((w+1)^{+}-1\right) \quad \text { in } \Omega \\
w=0, \quad \Delta w=0, \quad \text { on } \partial \Omega .
\end{gathered}
$$

Any element $u \in L^{2}(\Omega)$ can be expressed by

$$
u=\sum h_{k} \phi_{k} \text { with } \sum h_{k}^{2}<\infty .
$$

Let $H$ be a subspace of $L^{2}(\Omega)$ defined by

$$
H=\left\{u \in L^{2}(\Omega)\left|\sum\right| \lambda_{k}\left(\lambda_{k}-c\right) \mid h_{k}^{2}<\infty\right\} .
$$

Then this is a complete normed space with a norm

$$
\|u\|=\left[\sum\left|\lambda_{k}\left(\lambda_{k}-c\right)\right| h_{k}^{2}\right]^{\frac{1}{2}}
$$

Since $\lambda_{k}\left(\lambda_{k}-c\right) \rightarrow+\infty$ and $c$ is fixed, we have

(i) $\Delta^{2} u+c \Delta u \in H$ implies $u \in H$.

(ii) $\|u\| \geq C\|u\|_{L^{2}(\Omega)}$, for some $C>0$.

(iii) $\|u\|_{L^{2}(\Omega)}=0$ if and only if $\|u\|=0$.

For the proof of the above results we refer [1].

LEMMA 2.1. Assume that $c$ is not an eigenvalue of $-\Delta, a+b \neq \lambda_{k}\left(\lambda_{k}-c\right)$ and bounded. Then all solutions in $L^{2}(\Omega)$ of

$$
\Delta^{2} w+c \Delta w=(a+b)\left((w+1)^{+}-1\right) \text { in } L^{2}(\Omega)
$$

belong to $H$.

Proof. Let us write $(a+b)\left((w+1)^{+}-1\right)=\sum h_{k} \varphi_{k} \in L^{2}(\Omega)$.

$$
\begin{gathered}
\left(\Delta^{2}+c \Delta\right)^{-1}(a+b)\left((w+1)^{+}-1\right)=\sum \frac{1}{\lambda_{k}\left(\lambda_{k}-c\right)} h_{k} \phi_{k} \in L^{2}(\Omega) . \\
\left\|\left(\Delta^{2}+c \Delta\right)^{-1}(a+b)\left((w+1)^{+}-1\right)\right\|=\sum\left|\lambda_{k}\left(\lambda_{k}-c\right)\right| \frac{1}{\left(\lambda_{k}\left(\lambda_{k}-c\right)\right)^{2}} h_{k}^{2} \\
\leq C \sum h_{k}^{2}=C\|w\|_{L^{2}(\omega)}^{2}<\infty
\end{gathered}
$$

for some $C>0$. Thus $\left(\Delta^{2}+c \Delta\right)^{-1}\left((a+b)\left((w+1)^{+}-1\right)\right) \in H$.

With the aid of Lemma 2.1 it is enough that we investigate the existence of the solutions of (1.1) in the subspace $H$ of $L^{2}(\Omega)$.

Let us define the functional

$$
F(w)=\int_{\Omega} \frac{1}{2}|\Delta w|^{2}-\frac{c}{2}|\nabla w|^{2}-\frac{a+b}{2}|w+1|^{+}-(a+b) w .
$$


If we assume that $\lambda_{k}<c<\lambda_{k+1}$ and $a+b$ is bounded, $F(u)$ is well defined. By the following lemma, $F(w) \in C^{1}$. Thus the critical points of the functional $F(w)$ coincide with the weak solutions of (2.2).

LEMMA 2.2. Assume that $\lambda_{k}<c<\lambda_{k+1}$ and $a+b$ is bounded. Then the functional $F$ $(w)$ is continuous and Frechét differentiable in $H$ and

$$
D F(w)(h)=\int_{\Omega}\left[\Delta w \cdot \Delta h-c \nabla w \cdot \nabla h-(a+b)(w+1)^{+} h-(a+b) h\right] d x
$$

for $h \in H$.

Proof. First we shall prove that $F(w)$ is continuous at $w$. Let $w, z \in H$.

$$
\begin{aligned}
& F(w+z)-F(w) \\
= & \int_{\Omega}\left[\frac{1}{2}|\Delta(w+z)|^{2}-\frac{c}{2}|\nabla(w+z)|^{2}-\frac{a+b}{2}\left|(w+z+1)^{+}\right|^{2}-(a+b)\right. \\
& (w+z)] d x-\int_{\Omega}\left[\frac{1}{2}|\Delta w|^{2}-\frac{c}{2}|\nabla w|^{2}-\frac{a+b}{2}\left|(w+1)^{+}\right|^{2}-(a+b) w\right] d x \\
= & \int_{\Omega}\left[w \cdot\left(\Delta^{2} z+c \Delta z\right)+\frac{1}{2} z \cdot\left(\Delta^{2} z+c \Delta z\right)-\left(\frac{a+b}{2}\left|(w+z+1)^{+}\right|^{2}\right.\right. \\
& \left.\left.-\frac{a+b}{2}\left|(w+1)^{+}\right|^{2}-(a+b) z\right)\right] d x .
\end{aligned}
$$

Let $w=\sum h_{k} \varphi_{k}, z=\sum \tilde{h}_{k} \phi_{k}$. Then we have

$$
\begin{gathered}
\left|\int_{\Omega} w \cdot\left(\Delta^{2} z+c \Delta z\right) d x\right|=\left|\sum \int_{\Omega} \lambda_{k}\left(\lambda_{k}-c\right) h_{k} \tilde{h}_{k}\right| \leq\|w\|\|z\|, \\
\left|\int_{\Omega} z \cdot\left(\Delta^{2} z+c \Delta z\right) d x\right|=\left|\sum \lambda_{k}\left(\lambda_{k}-c\right) \tilde{h}_{k}^{2}\right| \leq\|z\|^{2} .
\end{gathered}
$$

On the other hand, by Mean Value Theorem, we have

$$
\left\|\frac{a+b}{2}\left|(w+z+1)^{+}\right|^{2}-\frac{a+b}{2}\left|(w+1)^{+}\right|^{2}\right\| \leq(a+b)\|z\| .
$$

Thus we have

$$
\left\|\frac{a+b}{2}\left|(w+z+1)^{+}\right|^{2}-\frac{a+b}{2}\left|(w+1)^{+}\right|^{2}-(a+b) z\right\| \leq 2(a+b)\|z\|=O(\|z\|) .
$$

Thus $F(w)$ is continuous at $w$. Next we shall prove that $F(w)$ is Fréchet differentiable at $w \in H$. We consider

$$
\begin{aligned}
& |F(w+z)-F(w)-D F(w) z|=\mid \int_{\Omega} \frac{1}{2} z\left(\Delta^{2} z+c \Delta z\right) \\
& -\left(\frac{a+b}{2}\left|(w+z+1)^{+}\right|^{2}-\frac{a+b}{2}\left|(w+1)^{+}\right|^{2}+(a+b)(w+1)^{+} z\right) \mid \\
& \leq \frac{1}{2}\|z\|^{2}+(a+b)\|z\|+(a+b)(\|w\|+1)\|z\| \\
& =\|z\|\left(\frac{1}{2}\|z\|+(a+b)+(a+b)(\|w\|+1)\right)=O(\|z\|) .
\end{aligned}
$$

Thus $F(w)$ is Fréchet differentiable at $w \in H$. 


\section{Proof of Theorem 1.1}

Throughout this section we assume that $\lambda_{k}<c<\lambda_{k+1}$ and $\lambda_{k+n}\left(\lambda_{k+n}-c\right)<a+b<\lambda_{k+n}$ ${ }_{+1}\left(\lambda_{k+n+1}-c\right)$. We shall prove Theorem 1.1 by applying the variation of linking method (cf. Theorem 4.2 of [8]). Now, we recall a variation of linking theorem of the existence of critical levels for a functional.

Let $X$ be an Hilbert space, $Y \subset X, \rho>0$ and $e \in X \backslash Y, e \neq 0$. Set:

$$
\begin{gathered}
B_{\rho}(Y)=\left\{x \in Y:\|x\|_{X} \leq \rho\right\}, \\
S_{\rho}(Y)=\left\{x \in Y:\|x\|_{X}=\rho\right\}, \\
\Delta_{\rho}(e, Y)=\left\{\sigma e+v: \sigma \geq 0, v \in Y,\|\sigma e+v\|_{X} \leq \rho\right\}, \\
\Sigma_{\rho}(e, Y)=\left\{\sigma e+v: \sigma \geq 0, v \in Y,\|\sigma e+v\|_{X}=\rho\right\} \cup\left\{v: v \in Y,\|v\|_{X} \leq \rho\right\} .
\end{gathered}
$$

THEOREM 3.1. ("A Variation of Linking") Let $\times$ be an Hilbert space, which is topological direct sum of the subspaces $X_{1}$ and $X_{2}$. Let $F \in C^{1}(X, R)$. Moreover assume:

(a) $\operatorname{dim} X_{1}<+\infty$;

(b) there exist $\rho>0, R>0$ and $e \in X_{1}, e \neq 0$ such that $\rho<R$ and

$$
\sup _{S_{\rho}\left(X_{1}\right)} F<\inf _{\Sigma_{R}\left(e, X_{2}\right)} F ;
$$

(c) $-\infty<a=\inf _{\Delta_{R}\left(e, X_{2}\right)} F$;

(d) $(P . S .)_{c}$ holds for any $c \in[a, b]$, where $b=\sup _{B_{\rho}\left(X_{1}\right)} F$.

Then there exist at least two critical levels $c_{1}$ and $c_{2}$ for the functional F such that:

$$
\inf _{\Delta_{R}\left(e, X_{2}\right)} F \leq c_{1} \leq \sup _{S_{\rho}\left(X_{1}\right)} F<\inf _{\Sigma_{R}\left(e, X_{2}\right)} F \leq c_{2} \leq \sup _{B_{\rho}\left(X_{1}\right)} F
$$

Let $H^{+}$be the subspace of $H$ spanned by the eigenfunctions corresponding to the eigenvalues $\lambda_{k}\left(\lambda_{k}-c\right)>0$ and $H^{-}$the subspace of $H$ spanned by the eigenfunctions corresponding to the eigenvalues $\lambda_{k}\left(\lambda_{k}-c\right)<0$. Then $H=H^{+} \oplus H$. Let $H_{k}$ be the subspace of $H$ spanned by $\varphi_{1}, \ldots, \varphi_{k}$ whose eigenvalues are $\lambda_{1}\left(\lambda_{1}-c\right), \ldots, \lambda_{k}\left(\lambda_{k}-c\right)$. Let $H_{k}^{\perp}$ be the orthogonal complement of $H_{k}$ in $H$. Then

$$
H=H_{k} \oplus H_{k}^{\perp} .
$$

Let $e \in H^{+} \cap H_{k+n} e \neq 0$ and $\rho>0$. Let us set

$$
\begin{gathered}
B_{\rho}\left(H_{k+n}\right)=\left\{w \in H_{k+n} \mid\|w\| \leq \rho\right\}, \\
S_{\rho}\left(H_{k+n}\right)=\left\{w \in H_{k+n} \mid\|w\|=\rho\right\}, \\
\Delta_{\rho}\left(e, H_{k+n}^{\perp}\right)=\left\{\sigma e+w \mid \sigma \geq 0, w \in H_{k+n}^{\perp}\|\sigma e+w\| \leq \rho\right\}, \\
\Sigma_{\rho}\left(e, H_{k+n}^{\perp}\right)=\left\{\sigma e+w \mid \sigma \geq 0, w \in H_{k+n^{\prime}}^{\perp}\|\sigma e+w\|=\rho\right\} \\
\cup\left\{w \mid w \in H_{k+n^{\prime}}^{\perp}\|w\| \leq \rho\right\} .
\end{gathered}
$$

Let $L: H \rightarrow H$ be the linear continuous operator such that

$$
(L w) z=\int_{\Omega}\left(\Delta^{2} w+c \Delta w\right) \cdot z d x-(a+b) \int_{\Omega} w z d x .
$$

Then $L$ is an isomorphism and $H_{k+n}, H_{k+n}^{\perp}$ are the negative space and the positive space of $L$. Thus we have 


$$
\begin{aligned}
& (L w) w \leq-\left((a+b)-\lambda_{k+n}\left(\lambda_{k+n}-c\right)\right)\|w\|^{2}, \quad w \in H_{k+n} \\
& (L w) w \geq\left(\lambda_{k+n+1}\left(\lambda_{k+n+1}-c\right)-(a+b)\right)\|w\|^{2}, \quad w \in H_{k+n}^{\perp}
\end{aligned}
$$

We can write

$$
F(w)=\frac{1}{2}(L w) w-\psi(w)
$$

where

$$
\psi(w)=\int_{\Omega} \frac{a+b}{2}\left|(w+1)^{-}\right|^{2} d x
$$

Since $H$ is compactly embedded in $L^{2}$, the map $D \psi: H \rightarrow H$ is compact.

LEMMA 3.1. Let $\lambda_{k}<c<\lambda_{k+1}$ and $\lambda_{k+n}\left(\lambda_{k+n}-c\right)<a+b<\lambda_{k+n+1}\left(\lambda_{k+n+1}-c\right)$. Then F(w) satisfies the (P.S. $)_{\gamma}$ condition for any $\gamma \in R$.

Proof. Let $\left(w_{n}\right)$ be a sequence in $H$ with $D F\left(w_{n}\right) \rightarrow 0$ and $F\left(w_{n}\right) \rightarrow \gamma$. Since $L$ is an isomorphism and $D \psi$ is compact, it is sufficent to show that $\left(w_{n}\right)$ is bounded in $H$. We argue by contradiction. we suppose that $\left\|w_{n}\right\| \rightarrow+\infty$. Let $z_{n}=\frac{w_{n}}{\left\|w_{n}\right\|}$. Up to a subsequence, we have $z_{n} \rightarrow z$ in $H$. Since $D F\left(w_{n}\right) \rightarrow 0$, we get

$$
\frac{D F\left(w_{n}\right) w_{n}}{\left\|w_{n}\right\|^{2}}=\int_{\Omega}\left(\Delta^{2}+c \Delta\right) z_{n}^{2}-\int_{\Omega}\left[(a+b)\left(z_{n}+\frac{1}{\left\|w_{n}\right\|}\right)^{+} z_{n}-(a+b) \frac{z_{n}}{\left\|w_{n}\right\|}\right] \rightarrow 0 .
$$

Let $P^{+}: H \rightarrow H_{k+n}^{\perp}$ and $P^{-}: H \rightarrow H_{k+n}$ denote the orthogonal projections. Since $P^{+} z_{n}-P^{-} z_{n}$ is bounded in $H$, we have

$$
\begin{aligned}
& \int_{\Omega}\left(\Delta^{2}+c \Delta\right)\left(P^{+} z_{n}+P^{-} z_{n}\right)\left(P^{+} z_{n}-P^{-} z_{n}\right) \\
& -\int_{\Omega}\left[(a+b)\left(P^{+} z_{n}+P^{-} z_{n}+\frac{1}{\left\|w_{n}\right\|}\right)^{+}\left(P^{+} z_{n}-P^{-} z_{n}\right)\right] \rightarrow 0 .
\end{aligned}
$$

Since $P^{+} z_{n}-P^{-} z_{n} \rightarrow P^{+} z-P^{-} z$ in $H$, from (3.2) and (3.3) we get

$$
0 \leq \int_{\Omega}\left[\left((a+b) z^{+}\right)\left(P^{+} z-P^{-} z\right)\right] d x \text {. }
$$

Hence $z \neq 0$. On the other hand, from (3.5), we get

$$
\begin{aligned}
0 & =\int_{\Omega}\left(\Delta^{2}+c \Delta\right)\left(P^{+} z+P^{-} z\right)\left(P^{+} z-P^{-} z\right)-\int_{\Omega}\left[(a+b) z^{+}\left(P^{+} z-P^{-} z\right)\right] \\
& \left.\geq \int_{\Omega}\left(\Delta^{2}+c \Delta\right)\left(P^{+} z+P^{-} z\right)\left(P^{+} z-P^{-} z\right)-\int_{\Omega}\left[(a+b) z\left(P^{+} z\right)-P^{-} z\right)\right] \\
& \left.\left.=\int_{\Omega}\left(\Delta^{2}+c \Delta\right)\left(P^{+} z+P^{-} z\right)\left(P^{+} z-P^{-} z\right)-\int_{\Omega}(a+b)\left(P^{+} z\right)+P^{-} z\right)\left(P^{+} z\right)-P^{-} z\right) \\
& =\int_{\Omega}\left(\Delta^{2}+c \Delta-(a+b)\right)\left(P^{+} z\right)^{2} d x-\int_{\Omega}\left(\Delta^{2}+c \Delta-(a+b)\right)\left(P^{-} z\right)^{2} \\
& \geq\left(\lambda_{k+n+1}\left(\lambda_{k+n+1}-c\right)-(a+b)\right)\left\|P^{+} z\right\|_{L^{\Omega}}^{2}-\left(\lambda_{k+n}\left(\lambda_{k+n}-c\right)-(a+b)\right)\left\|P^{-} z\right\|_{L^{2}(\Omega)}^{2} .
\end{aligned}
$$

The last line of (3.6) is positive or equal to 0 since $\lambda_{k+n+1}\left(\lambda_{k+n+1}-c\right)-(a+b)>0$ and $-\left(\lambda_{k+n}\left(\lambda_{k+n}-c\right)-(a+b)\right)>0$. Thus the only possibility to hold (3.6) is that $P^{+} z=$ 0 and $P^{-} z=0$. Thus $z=0$, which gives a contradiction.

LEMMA 3.2. Let $\lambda_{k}<c<\lambda_{k+1}$ and $\lambda_{k+n}\left(\lambda_{k+n}-c\right)<b<\lambda_{k+n+1}\left(\lambda_{k+n+1}-c\right)$. 


\section{Then}

(i) there exists $R_{k+n}>0$ such that the functional $F(w)$ is bounded from below on $H_{k+n}^{\perp}$;

$$
\inf _{\substack{w \in H_{k+n}^{\perp} \\\|w\|=R_{k+n}}} F(w)>0 \text { and } \inf _{\substack{w \in H_{k+n}^{\perp} \\\|w\| R_{k+n}}} F(w)>-\infty .
$$

(ii) there exists $\rho_{k+n}>0$ such that

$$
\sup _{\substack{w \in H_{k+n} \\\|w\|=\rho_{k+n}}} F(w)<0 \text { and } \sup _{\substack{w \in H_{k+n} \\\|w\| \leq \rho_{k+n}}} F(w)<\infty .
$$

Proof. (i) Let $w \in H_{k+n}^{\perp}$. Then we have

$$
\begin{aligned}
& \lim _{\substack{w \in H_{k+n}^{+} \\
\|w\| \rightarrow+\infty}} F(w) \geq \lim _{\substack{w \in H_{k+n}^{+} \\
\|w\|_{k+n} \rightarrow \infty}} \frac{1}{2}\left(1-\frac{r}{\lambda_{k+n+1}\left(\lambda_{k+n+1}-c\right)}\right)\|w\|^{2} \\
& -\lim _{\substack{w \in H_{k+n}^{+} \\
\|w\| \rightarrow+\infty}} \int_{\Omega}\left[\frac{a+b}{2}\left|(w+1)^{+}\right|^{2}-(a+b) w-\frac{r}{2} w^{2}\right] d x \\
& \geq \lim _{\substack{w \in H_{k+n}^{+} \\
\|w\| \rightarrow \infty}} \frac{1}{2}\left(1-\frac{r}{\lambda_{k+n+1}\left(\lambda_{k+n+1}-c\right)}\right)\|w\|^{2}-\lim _{\substack{w \in H_{k+n}^{+} \\
\|w\| \rightarrow+\infty}} \int_{\Omega}\left[\frac{a+b}{2}\left(w^{2}+1\right)-\frac{r}{2} w^{2}\right] d x \\
& \geq \lim _{\substack{w \in H_{k+n}^{+} \\
\|w\| \rightarrow+\infty}} \frac{1}{2}\left(1-\frac{r}{\lambda_{k+n+1}\left(\lambda_{k+n+1}-c\right)}\right)\|w\|^{2}-\lim _{\substack{w \in H_{k+n}^{+} \\
\|w\| \rightarrow+\infty}} \frac{1}{2}((a+b)-r) \int_{\Omega} w^{2} \\
& -\frac{a+b}{2}|\Omega| \rightarrow+\infty,
\end{aligned}
$$

since $a+b-r<\lambda_{k+n+1}\left(\lambda_{k+n+1}-c\right)-r=\frac{\lambda_{k+n+1}\left(\lambda_{k+n+1}-c\right)-\lambda_{k+n}\left(\lambda_{k+n}-c\right)}{2}$. Thus there exists $R_{k+n}>0$ such that $\inf _{\substack{w \in H_{k+n} \\\|w\|=R_{k+n}}} F(w)>0$. Moreover if $w \in H_{k+n}^{\perp}$ with $\|w\|<R_{k+n}$, then we have

$$
\begin{aligned}
F(w) & \geq \frac{1}{2}\left(\lambda_{k+n+1}\left(\lambda_{k+n+1}-c\right)\right)\|w\|_{L^{2}(\Omega)}^{2}-\int_{\Omega}\left[\frac{a+b}{2}(w+1)^{2}--(a+b) w\right] d x \\
& >\frac{1}{2}\left\{\left(\lambda_{k+n+1}\left(\lambda_{k+n+1}-c\right)\right)-(a+b)\right\}\|w\|_{L^{2}(\Omega)}^{2}-\int_{\Omega} \frac{a+b}{2} d x>-\infty .
\end{aligned}
$$

Thus we have $\inf _{\substack{w \in H_{k+n}^{\perp} \\\|w\|<R_{k+n}}} F(w)>--\infty$.

(ii) Let $w \in H_{k+n}$. Then

$$
(L w) w \leq\left(\lambda_{k+n}\left(\lambda_{k+n}-c\right)-r\right) \int_{\Omega} w^{2} d x \leq \frac{\lambda_{k+n}\left(\lambda_{k+n}-c\right)-\lambda_{k+n+1}\left(\lambda_{k+n+1}-c\right)}{2} \int_{\Omega} w^{+2},
$$$$
\int_{\Omega}\left[\frac{1}{2}(a+b)\left|(w+1)^{+}\right|^{2}-(a+b) w-r w^{2}\right] d x \geq \int_{\Omega}\left[\frac{1}{2}(a+b)\left|w^{+}\right|^{2}-(a+b) w-r w^{+2}\right] d x,
$$

so that

$$
\begin{aligned}
F(w) \leq & \frac{1}{2} \frac{\lambda_{k+n}\left(\lambda_{k+n}-c\right)-\lambda_{k+n+1}\left(\lambda_{k+n+1}-c\right)}{2} \int_{\Omega} w^{+2} \\
& -\frac{a+b-r}{2} \int_{\Omega} w^{+2}+\int_{\Omega}(a+b) w d x \\
\leq & \frac{1}{2}\left\{\frac{\lambda_{k+n}\left(\lambda_{k+n}-c\right)-\lambda_{k+n+1}\left(\lambda_{k+n+1}-c\right)}{2}-(a+b-r)\right\}\left\|w^{+}\right\|_{L^{2}(\Omega)}^{2} \\
& +(a+b)\|w\|_{L^{2}(\Omega)} .
\end{aligned}
$$


Since $\frac{\lambda_{k+n}\left(\lambda_{k+n}-c\right)-\lambda_{k+n+1}\left(\lambda_{k+n+1}-c\right)}{2}-(a+b-r)<0$, there exists $\rho_{k+n}>0$ such that if $w \in$ $H_{k+n}$ and $\|w\|=\rho_{k+n}$, then $\sup F(w)<0$. Moreover, if $w \in H_{k+n}$ and $\|w\| \leq \rho_{k+n}$, then

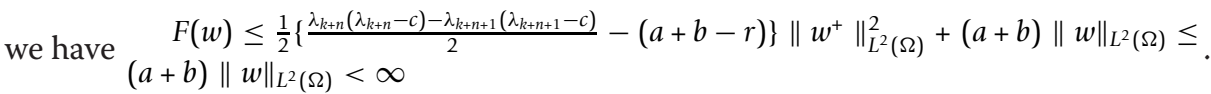

LEMMA 3.3. Let $\lambda_{k}<c<\lambda_{k+1}, \lambda_{k+n}\left(\lambda_{k+n}-c\right)<a+b<\lambda_{k+n+1}\left(\lambda_{k+n+1}-c\right)$ and let $e_{1} \in$ $H^{+} \cap H_{k+n}$ with $\left\|e_{1}\right\|=1$. Then there exists $R_{k+n}^{-}$such that $R_{k+n}^{-}>\rho_{k+n}$,

$$
\inf _{w \in \Sigma_{R_{k+n}^{-}}\left(e_{1}, H_{k+n}^{\perp}\right)} F(w) \geq 0 \text { and } \inf _{w \in \Delta_{R_{k+n}^{-}}\left(e_{1}, H_{k+n}^{\perp}\right)} F(w) \geq-\infty .
$$

Proof. Let us chose $w \in H_{k+n}^{\perp}$ and $\sigma \geq 0$ and $e_{1} \in H^{+} \cap H_{k+n}$ with $\left\|e_{1}\right\|=1$. Then we get

$$
\begin{aligned}
F\left(w+\sigma e_{1}\right) \geq & \frac{1}{2} \lambda_{k+n+1}\left(\lambda_{k+n+1}-c\right)\|w\|_{L^{2}(\Omega)}^{2}+\frac{\sigma^{2}}{2}\left\|e_{1}\right\|^{2} \\
& -\int_{\Omega}\left[\frac{a+b}{2}\left(w+\sigma e_{1}+1\right)^{2}-(a+b)\left(w+\sigma e_{1}\right)\right] d x \\
= & \frac{1}{2}\left\{\lambda_{k+n+1}\left(\lambda_{k+n+1}-c\right)-(a+b)\right\}\|w\|_{L^{2}(\Omega)}^{2}+\frac{\sigma^{2}}{2}(\Lambda-(a+b))\left\|e_{1}\right\|_{L^{2}(\Omega)}^{2} \\
& -(a+b) \sigma^{2}\|w\|_{L^{2}(\Omega)}\left\|e_{1}\right\|_{L^{2}(\Omega)}-\frac{a+b}{2}|\Omega|
\end{aligned}
$$

where $\lambda_{k+1}\left(\lambda_{k+1}-c\right) \leq \Lambda \leq \lambda_{k+1}\left(\lambda_{k+1}-c\right)$. Choose $\sigma>0$ so mall that $\frac{\sigma}{2}\left\|e_{1}\right\|^{2}$ is small. We can choose a number $R_{k+n}^{-}>0$ such that $R_{k+n}^{-}>\sigma, R_{k+n}^{-}>\rho_{k+n}$, and $\inf _{\substack{w \in H_{k+n_{1},}^{\perp}, \sigma \geq 0 \\\left\|w+\sigma e_{1}\right\|=R_{k+n}}} F\left(w+\sigma e_{1}\right) \geq 0$ : Moreover if $w \in H_{k+n^{\prime}}^{\perp} \sigma \geq 0\left\|w+\sigma e_{1}\right\| \leq R_{k+n}^{-}$, then $F(w) \geq \frac{\sigma^{2}}{2}(\Lambda-b)\left\|e_{1}\right\|_{L^{2}(\Omega)}^{2}-(a+b) \sigma\|w\|_{L^{2}(\Omega)}\left\|e_{1}\right\|_{L^{2}(\Omega)}-\frac{a+b}{2}|\Omega| \geq-\infty$. Thus we prove the lemma.

\section{Proof of Theorem 1.1}

By Lemma 2.2, $F(w)$ is continuous and Frechét differentiable in H. By Lemma 3.1. $F(w)$ satisfies the (P.S.) $)_{\gamma}$ condition for any $\gamma \in R$. We note that the subspace $S_{\rho_{k+n}} \cap H_{k+n}$ and the subspace $\Sigma_{R_{k+n}^{-}}\left(e_{1}, H_{k+n}^{\perp}\right)$ link at the subspace $\left\{e_{1}\right\}$. By Lemma 3.2 and Lemma 3.3, we have

$$
\sup _{w \in S_{\rho_{k+n}} \cap H_{k+n}} F(w)<\inf _{w \in \Sigma_{R_{k+n}^{-}}\left(e_{1}, H_{k+n}^{\perp}\right)} F(w) .
$$

By Lemma 3.3, we also have $\inf _{w \in \Delta_{R_{k+n}^{-}}\left(e_{1}, H_{k+n}^{\perp}\right)} F(w)>-\infty$ Thus by the variation of linking theorem, there exists at least two nontrivial solutions of (2.2). Thus we complete the Theorem 1.1.

\section{Proof of Theorem 1.2}

\section{Proof of Theorem 1.2}

Assume that $\lambda_{k}<c<\lambda_{k+1}$ and $\lambda_{k}\left(\lambda_{k}-c\right)<0, b<\lambda_{k+1}\left(\lambda_{k+1}-c\right)$. Let $r=\frac{1}{2}\left\{\lambda_{k}\left(\lambda_{k}-c\right)+\lambda_{k+1}\left(\lambda_{k+1}-c\right)\right\}$. We can rewrite (2.2) as

$$
\begin{gathered}
\left(\Delta^{2}+c \Delta-r\right) w=(a+b)(w+1)^{+}-r(w+1)^{+}+r(w+1)^{+}-r w-(a+b) \text { in } L^{2}(\Omega), \\
w=0, \quad \Delta w=0 \quad \text { on } \partial \Omega .
\end{gathered}
$$


or

$$
\begin{gathered}
w=\left(\Delta^{2}+c \Delta-r\right)^{-1}\left[(a+b)(w+1)^{+}-r(w+1)^{+}+r(w+1)^{+}-r w-(a+b)\right] \text { in } L^{2}(\Omega) \\
w=0, \quad \Delta w=0 \quad \text { on } \partial \Omega .
\end{gathered}
$$

We note that the operator $\left(\Delta^{2}+c \Delta-r\right)^{-1}$ is a compact, self-adjoint and linear map from $L^{2}(\Omega)$ into $L^{2}(\Omega)$ with norm $\frac{2}{\lambda_{k+1}\left(\lambda_{k+1}-c\right)-\lambda_{k}\left(\lambda_{k}-c\right)}$, and

$$
\begin{aligned}
& \left\|((a+b)-r)\left\{\left(w_{2}+1\right)^{+}-\left(w_{1}+1\right)^{+}\right\}+r\left\{\left(w_{2}+1\right)^{+}-\left(w_{1}+1\right)^{+}\right\}-r\left(w_{2}-w_{1}\right)\right\| \\
& \quad \leq \max \{(a+b)-r, r\}\left\|w_{2}-w_{1}\right\|<\frac{1}{2}\left\{\lambda_{k+1}\left(\lambda_{k+1}-c\right)-\lambda_{k}\left(\lambda_{k}-c\right)\right\}\left\|w_{2}-w_{1}\right\| .
\end{aligned}
$$

Thus the right hand side of (4.2) defines a Lipschitz mapping from $L^{2}(\Omega)$ into $L^{2}(\Omega)$ with Lipschitz constant $<1$. By the contraction mapping principle, there exists a unique solution $w \in L^{2}(\Omega)$ of (4.2). By Lemma 2.1, the solution $u \in H$. We complete the proof.

\section{Abbreviations}

(FESDBC): fourth-order elliptic system with Dirichlet boundary condition.

\section{Acknowledgements}

This work(Tacksun Jung) was supported by Basic Science Research Program through the National Research Foundation of Korea(NRF) funded by the Ministry of Education, Science and Technology (KRF-2010-0023985).

\section{Author details}

${ }^{1}$ Department of Mathematics, Kunsan National University, Kunsan 573-701, Korea ${ }^{2}$ Department of Mathematics Education, Inha University, Incheon 402-751, Korea

\section{Authors' contributions}

TJ carried out (FESDBC) studies, participated in the sequence alignment and drafted the manuscript. QC participated in the sequence alignment. All authors read and approved the final manuscript.

\section{Competing interests}

The authors declare that they have no competing interests.

Received: 13 February 2011 Accepted: 17 September 2011 Published: 17 September 2011

\section{References}

1. Choi, QH, Jung, T: Multiplicity of solutions and source terms in a fourth order nonlinear elliptic equation. Acta Mathematica Scientia. 19(4), 361-374 (1999)

2. Choi, QH, Jung, T: Multiplicity results on nonlinear biharmonic operator. Rocky Mountain J Math. 29(1), 141-164 (1999). doi:10.1216/rmjm/1181071683

3. Jung, TS, Choi, QH: Multiplicity results on a nonlinear biharmonic equation. Nonlinear Analysis, Theory, Methods and Applications. 30(8), 5083-5092 (1997). doi:10.1016/S0362-546X(97)00381-7

4. Jung, T, Choi, $\mathrm{QH}$ : On the existence of the third solution of the nonlinear biharmonic equation with Dirichlet boundary condition. Chungcheong Math J. 20, 81-94 (2007)

5. Lazer, AC, McKenna, PJ: Multiplicity results for a class of semilinear elliptic and parabolic boundary value problems. J Math Anal Appl. 107, 371-395 (1985). doi:10.1016/0022-247X(85)90320-8

6. Lazer, AC, McKenna, PJ: Large amplitude periodic oscillations in suspension bridges:, some new connections with nonlinear analysis. SIAM Review. 32, 537-578 (1990). doi:10.1137/1032120

7. McKenna, PJ, Walter, W: On the multiplicity of the solution set of some nonlinear boundary value problems. Nonlinear Analysis, Theory, Methods and Applications. 8, 893-907 (1984). doi:10.1016/0362-546X(84)90110-X

8. Micheletti, AM, Pistoia, A: Multiplicity results for a fourth-order semilinear elliptic problem. Nonlinear Analysis TMA. 31(7), 895-908 (1998). doi:10.1016/S0362-546X(97)00446-X

9. Micheletti, AM, Pistoia, A, Sacon, A: Three solutions of a 4th order elliptic problem via variational theorems of mixed type. Applicable analysis. 75, 43-59 (2000). doi:10.1080/00036810008840834

10. Tarantello, : A note on a semilinear elliptic problem. Diff Integ Equat. 5(3), 561-565 (1992)

doi:10.1186/1029-242X-2011-60

Cite this article as: Jung and Choi: Fourth order elliptic system with dirichlet boundary condition. Journal of Inequalities and Applications 2011 2011:60. 